\title{
Effects of ID-CBT5101 in Preventing and Alleviating Osteoarthritis Symptoms in a Monosodium lodoacetate-Induced Rat Model
}

\author{
Boo-Yong Sim ${ }^{1}$, Hak-Joo Choi ${ }^{1}$, Min-Goo Kim ${ }^{2}$, Dong-Gu Jeong ${ }^{2}$, Don-Gil Lee ${ }^{2}$, Jong-Min Yoon ${ }^{2}$, Dae-Jung Kang ${ }^{2}$, \\ Soobong Park ${ }^{2}$, Joong-Gu Ji ${ }^{3}$, In-Hwan Joo ${ }^{4}$, and Dong-Hee Kim ${ }^{1,4 *}$ \\ ${ }^{1}$ Traditional and Biomedical Research Center, Daejeon University, Daejeon 34520, Republic of Korea \\ ${ }^{2}$ Research Laboratories, Ildong Pharmaceutical Company, Gyeonggi-do 17575, Republic of Korea \\ ${ }^{3}$ Department of Oriental Health Care, Joongbu University, Chungcheongnam-do 32713, Republic of Korea \\ ${ }^{4}$ Department of Pathology, College of Oriental Medicine, Daejeon University, Daejeon 34520, Republic of Korea
}

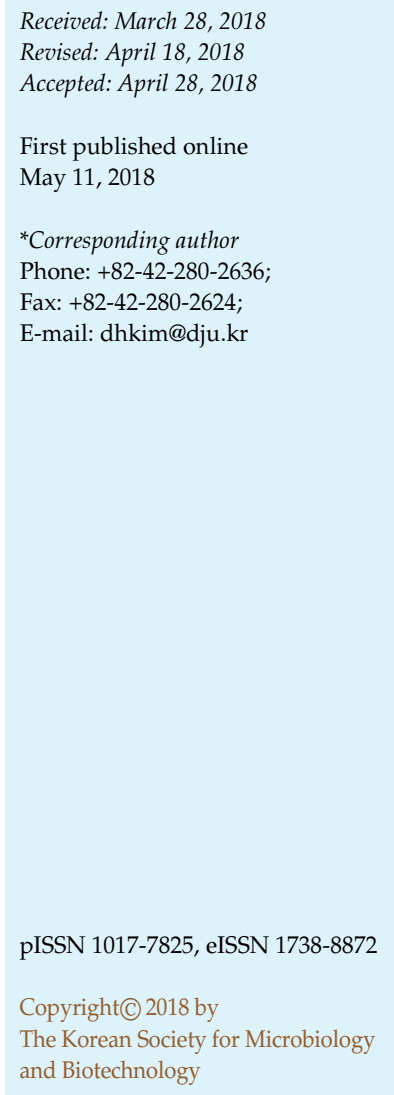

Osteoarthritis is a disease that affects the articular cartilage and osseous tissue, and can be worsened by aging, overweight status, and post-traumatic arthritis. The present study aimed to evaluate the effect of ID-CBT5101 (tyndallized Clostridium butyricum) on bone metabolism and the inflammatory response in a monosodium iodoacetate-induced rat model of osteoarthritis. ID-CBT5101 was administered orally at doses of $10^{8}$ or $10^{10} \mathrm{CFU} /$ day for 2 weeks before direct injection of monosodium iodoacetate ( $3 \mathrm{mg} / 50 \mu \mathrm{l}$ of $0.9 \%$ saline) into the intra-articular space of the rats' right knees. The rats subsequently received the same doses of oral ID-CBT5101 for another 4 weeks. We evaluated the treatment effects based on serum biomarkers, mRNA expression, morphological and histopathological analyses of the knee joints, and weight-bearing distribution analysis. Compared with those in control rats, the IDCBT5101 treatments significantly reduced the serum concentration of inflammation and bone metabolism markers (i.e., COX-2, IL-6, $\mathrm{LTB}_{4}$, and COMP), and significantly increased the concentration of IFN- $\gamma$ and glycosaminoglycans. In addition, the ID-CBT5101 treatments inhibited the mRNA expression of matrix metalloproteinases and tissue inhibitors of metalloproteinases (i.e., MMP-2, MMP-3, MMP-9, MMP-13, TIMP-1, and TIMP-2). Furthermore, the ID-CBT5101 treatments effectively preserved the knee cartilage and synovial membrane, and significantly decreased the amount of fibrous tissue. Moreover, compared with that of the negative control group, the ID-CBT5101 treatments increased the weight-bearing distribution by $\geq 20 \%$. The results indicate that ID-CBT5101 prevented and alleviated osteoarthritis symptoms. Thus, ID-CBT5101 may be a novel therapeutic option for the management of osteoarthritis.

Keywords: Osteoarthritis, bone metabolism, inflammation, probiotics, cartilage degeneration

\section{Introduction}

Osteoarthritis (OA) is an inflammatory disease that mainly affects the joint cartilage and nearby bone tissue [1]. OA can be worsened by genetic factors, aging, overweight status, and post-traumatic arthritis [2, 3]. In cases of OA, hypofunction of the cartilage and joint leads to a limited range of joint motion, swelling, and pain [4], which are generally treated using pharmaceutical drugs (e.g., anti- inflammatory agents, cartilage protectants, and nonsteroidal anti-inflammatory drugs) or replacement arthroplasty. However, long-term drug treatment is associated with adverse effects on the gastrointestinal and/or cardiovascular systems [5]. To mimic the symptoms of OA and develop new treatments, researchers have created a monosodium iodoacetate (MIA)-induced rat model of human OA. The MIA experimental model involves destruction of the articular cartilage by inducing continuous infiltration of 
synoviocytes and inflammatory cells and is a commonly used animal model for evaluating pain associated with OA [6-8]. Thus, the MIA model has been widely used for the pharmacological evaluation of new drug therapies [9].

Probiotics are microorganisms that provide beneficial effects by improving the host's intestinal microflora [10, 11]. Clostridium butyricum is a probiotic butyric acid-producing bacterium that helps resolve diarrhea, constipation, abdominal bloating, and intestinal fermentation [12]. A previous study has also indicated that butyric acid (butyrate) produced by $C$. butyricum can help alleviate arthritis symptoms through intestinal immune regulation [13]. C. butyricum IDCC 5101 (ID-CBT5101; Ildong Pharmaceuticals) has been subjected to tyndallization, which is the firstknown method for sterilizing spore-forming bacteria by repeated boiling and incubation in enriched media. The present study aimed to evaluate the ability of ID-CBT5101 to prevent and alleviate OA symptoms in the MIA-induced rat model based on mRNA expression, morphological and histological analyses, weight-bearing analysis, and factors that are associated with inflammation and bone mineral metabolism.

\section{Materials and Methods}

\section{ID-CBT5101}

C. butyricum IDCC 5101 strain ID-CBT5101 was provided by the Ildong Pharmaceutical Research and Development Center (Ildong Pharmaceutical Co., Korea). The strain was cultured at $43^{\circ} \mathrm{C}$ for $16 \mathrm{~h}$ and the organisms were killed by heating at $121^{\circ} \mathrm{C}$ for $1 \mathrm{~h}$. The cultured media were then concentrated 5-fold using a rotary vacuum evaporator (N-1100; EYELA, Japan), and the concentrates were lyophilized. The present study used the lyophilized IDCBT5101 powder, which was diluted using corn starch powder.

\footnotetext{
Animals

Sixty male Sprague-Dawley rats (170-200 g) were obtained from RaonBio Inc. (Korea). The rats were acclimated for 10 days and housed under controlled conditions at a temperature of $22 \pm 2^{\circ} \mathrm{C}$, humidity of $55 \pm 15 \%$, a $12 \mathrm{~h}$ light/12 h dark cycle, and fresh-air ventilation (10-15 times/h). The rats were housed in solid-bottom cages with free access to food and water. All experimental procedures complied with the National Institute of Health Guide for the Care and Use of Laboratory Animals and the Korean National Animal Welfare Law. The experimental animal facility and study protocols were approved by the Institutional Animal Care and Use Committee of Daejeon University (DJUARB2016027). Written consent was obtained from the human participant who provided the samples. No human studies were conducted and hence ethical approval for the human sample was not applicable.
}

\section{Experimental Protocol}

ID-CBT5101 was administered orally for 2 weeks before the MIA solution ( $3 \mathrm{mg} / 50 \mu \mathrm{l}$ of $0.9 \%$ saline) was injected directly into the intra-articular space of the rats' right knees. This step was performed under anesthesia that was induced using an intraperitoneal injection of ketamine and xylazine [14]. The MIA solution was injected using a 31-gauge ultra-fine insulin syringe (Becton Dickinson, USA) that was inserted approximately 2-3 mm deep. After the injection of MIA, the rats with MIA-induced osteoarthritis received the same doses of ID-CBT5101 for 4 weeks.

Before starting the experiment, the rats were randomized to a normal control group (NC group, $n=10$ ), a positive control group (indomethacin (IM)-treated group, $n=10$ ), an OA control group (OAC group, $n=10$ ), or ID-CBT5101 treatment groups that received $10^{8} \mathrm{CFU} /$ day $(n=10)$ or $10^{10} \mathrm{CFU} /$ day $(n=10)$. The NC group received an equivalent volume of saline instead of MIA. The treatment groups received daily oral doses of ID-CBT5101 for a total of 6 weeks. The positive control group received oral IM at a dose of $2 \mathrm{mg} / \mathrm{kg} /$ day from 1 week after the MIA injection until the end of the experiment. The OAC group received oral distilled water. The administration volumes were $2 \mathrm{ml} /$ animal for all experimental groups.

\section{Measuring Serum Cytokines and Bone Metabolism Markers}

Serum levels of cyclooxygenase (COX)-2, interleukin (IL)-6, leukotriene B4 $\left(\mathrm{LTB}_{4}\right)$, interferon-gamma (IFN- $\gamma$ ), glycosaminoglycans (GAGs), and cartilage oligomeric matrix protein (COMP) were measured using commercial MILLIPLEX Multiplex Assay kits and enzyme-linked immunosorbent assay (ELISA) kits (IL-6 and IFN- $\gamma$, Merck Millipore, USA; $\mathrm{LTB}_{4}$ and TGF- $\beta$, R\&D Systems, USA; COX-2, COMP, and GAGs, Mybiosource, USA). All assays and procedures were performed according to the manufacturers' protocols.

\section{Quantitative Real-Time Polymerase Chain Reaction Analysis}

The samples for RNA extraction were obtained from the cartilage tissue of rats by cryogenic grinding, and total RNA was extracted using the Total RNA purification kit (Intron, Korea) according to the manufacturer's instructions. We transcribed $1 \mu \mathrm{g}$ of total RNA in a volume of $20 \mu \mathrm{l}$ using oligo(dT) primers, with the enzyme and buffer supplied in the Prime Script II 1st strand cDNA Synthesis kit (Takara, Japan). Quantitative real-time polymerase chain reactions (PCR) were performed using the MX3005P device (Stratagene, USA) and the primers that are shown in Table 1. SYBR Premix Ex Taq II (Takara, Japan) was used for the real-time PCR. The final volume of the reaction mixture was $25 \mu \mathrm{l}$, including $2 \mu \mathrm{l}$ of cDNA template, $12.5 \mu \mathrm{l}$ of Master Mix, $1 \mu \mathrm{l}$ of each primer (10 $\mu \mathrm{M}$ stock solution), and $8.5 \mu \mathrm{l}$ of sterile distilled water. The thermal cycling profile consisted of a pre-incubation step at $95^{\circ} \mathrm{C}$ for $10 \mathrm{~min}$, followed by 40 cycles at $95^{\circ} \mathrm{C}(15 \mathrm{sec})$ and $60^{\circ} \mathrm{C}(60 \mathrm{sec})$.

Relative quantitative evaluation of matrix metalloproteinases (MMP-2, MMP-3, MMP-9, and MMP-13) and tissue inhibitors of 
Table 1. Primer sequences used in the real-time polymerase chain reaction.

\begin{tabular}{cl}
\hline $\begin{array}{c}\text { Gene } \\
\text { name }\end{array}$ & \multicolumn{1}{c}{ Primer sequences } \\
\hline GAPDH & 5'-GCTTAAGAGACAGCCGCATCT-3' (sense) \\
& 5'-CGACCTTCACCATTTTGTCTACA-3' (antisense) \\
MMP-2 & 5'-TCCCGAGATCTGCAAGCAAG-3' (sense) \\
& 5'-AGAATGTGGCCACCAGCAAG-3' (antisense) \\
MMP-3 & 5'-TGATGGGCCTGGAATGGTC-3' (sense) \\
& 5'-TTCATGAGCAGCAACCAGGAATAG-3' (antisense) \\
MMP-9 & 5'-AGCCGGGAACGTATCTGGA-3' (sense) \\
& 5'-TGGAAACTCACACGCCAGAAG-3' (antisense) \\
MMP-13 & 5'-CCCTGGAATTGGCGACAAAG-3' (sense) \\
& 5'-GCATGACTCTCACAATGCGATTAC-3' (antisense) \\
TIMP-1 & 5'-CATCTCTGGCCTCTGGCATC-3' (sense) \\
& 5'-CATAACGCTGGTATAAGGTGGTCTC-3' (antisense) \\
TIMP-2 & 5'-GACACGCTTAGCATCACCCAGA-3' (sense) \\
& 5'-CTGTGACCCAGTCCATCCAGAG-3' (antisense)
\end{tabular}

GAPDH, glyceraldehyde 3-phosphate dehydrogenase; MMPs, matrix metalloproteinases; TIMPs, tissue inhibitor of metalloproteinases.

metalloproteinases (TIMP-1 and TIMP-2) was performed by comparing the cycle thresholds (CT) relative to the value for glyceraldehyde-3-phosphate dehydrogenase. The CTs were determined at the intersection between the amplification plot and the threshold.

\section{Measuring Hind Paw Weight-Bearing Distribution}

The MIA model causes disruption of the balance in the rats' hind paw weight-bearing capabilities. Thus, an incapacitance tester (Linton Instrumentation, UK) was used to evaluate changes in the hind paws' weight-bearing capabilities. The rats were carefully placed in a measuring chamber, and the weight-bearing force exerted on the hind limbs was averaged over a 10-sec period. The weight distribution ratio was calculated as (weight on the arthritic right hind limb / weight on the normal left hind limb) $\times 100$ [15]

\section{Histological Analysis}

Tissue specimens from the rats' right knee joints were removed, fixed in $10 \%$ formalin, calcified, embedded in paraffin, and serially sectioned to a thickness of $7 \mu \mathrm{m}$. The tissue sections were then stained using hematoxylin and eosin (H\&E) or Safranin O. Histological changes were examined using a light microscope (Axioskop 40; Carl Zeiss AG, Germany) and photographed (Axiocam MRc5; Carl Zeiss AG, Germany). The severity of OA was evaluated in the medial compartment of the knee using the Osteoarthritis Research Society International (OARSI) scoring system after staining with H\&E and Safranin O [16]. The cartilage degeneration was scored using a scoring system that measured the medial tibia plateau on a scale of $0-5(0=$ no degeneration, $1=$ mild degeneration in the surface, 2 = slightly extended degeneration in the upper center, $3=$ moderate degeneration in the median area, $4=$ extended deep degeneration, and $5=$ severe degeneration).

\section{Morphological Analysis of Knee Cartilage}

The microarchitecture of the knee joints at $0.6-2.1 \mathrm{~mm}$ from the growth plate of the distal femur was scanned using a microcomputed tomography (micro-CT) system (Sky-Scan 1076; SkyScan, Belgium). The X-ray source was set at a voltage of $50 \mathrm{kV}$ and a current of $200 \mu \mathrm{A}$, and the beam was filtered using a $0.5-\mathrm{mm}$ aluminum filter. The scanning angular rotation was $180^{\circ}$ with an angular step of $0.5^{\circ}$ [17]. The voxel size was fixed at $8.9 \mu \mathrm{m}$. The morphometric indices of the bone region were determined using CTAn software (SkyScan 1076; SkyScan, Belgium).

\section{Data Analysis}

All results are presented as the mean \pm standard deviation. The statistical analyses were performed using one-way analysis of variance or Student's $t$-test, as appropriate.

\section{Results}

\section{Effects of ID-CBT5101 on Serum Levels of Inflammatory Factors}

Inflammatory factors help maintain chronic inflammation and tissue damage during the progression of OA [18]. Therefore, we investigated the effects of ID-CBT5101 on serum levels of IL-6, COX-2, and $\mathrm{LTB}_{4}$ in the MIA-induced rat $\mathrm{OA}$ model. Compared with the NC group, the OAC group had an elevated serum level of IL-6 and $\mathrm{LTB}_{4}$. Compared with the OAC group, the ID-CBT5101 treatment and IM treatment groups exhibited significantly decreased serum levels of IL-6 and $\mathrm{LTB}_{4}(p<0.05)$ (Figs. 1A and 1B). However, the serum concentration of COX-2 decreased significantly only in the group that received $10^{10} \mathrm{CFU} /$ day of ID-CBT5101 $(p<0.05)$ (Fig. 1C). These results indicate that ID-CBT5101 might have anti-inflammatory effects by inhibiting inflammatory factors in the rat OA model.

\section{Effects of ID-CBT5101 on Serum Levels of Bone Metabolism Factors}

Bone metabolism factors can help prevent cartilage damage in cases of OA, and IFN- $\gamma$, GAGs, and COMP are well-known biomarkers for bone/cartilage production or damage [19-22]. Therefore, we investigated the effects of ID-CBT5101 on the serum level of IFN- $\gamma$, GAGs, and COMP in the MIA-induced OA model. There were no significant differences in the serum level of IFN- $\gamma$, GAGs, and COMP among the NC-, OAC-, and IM-treated groups. The group 

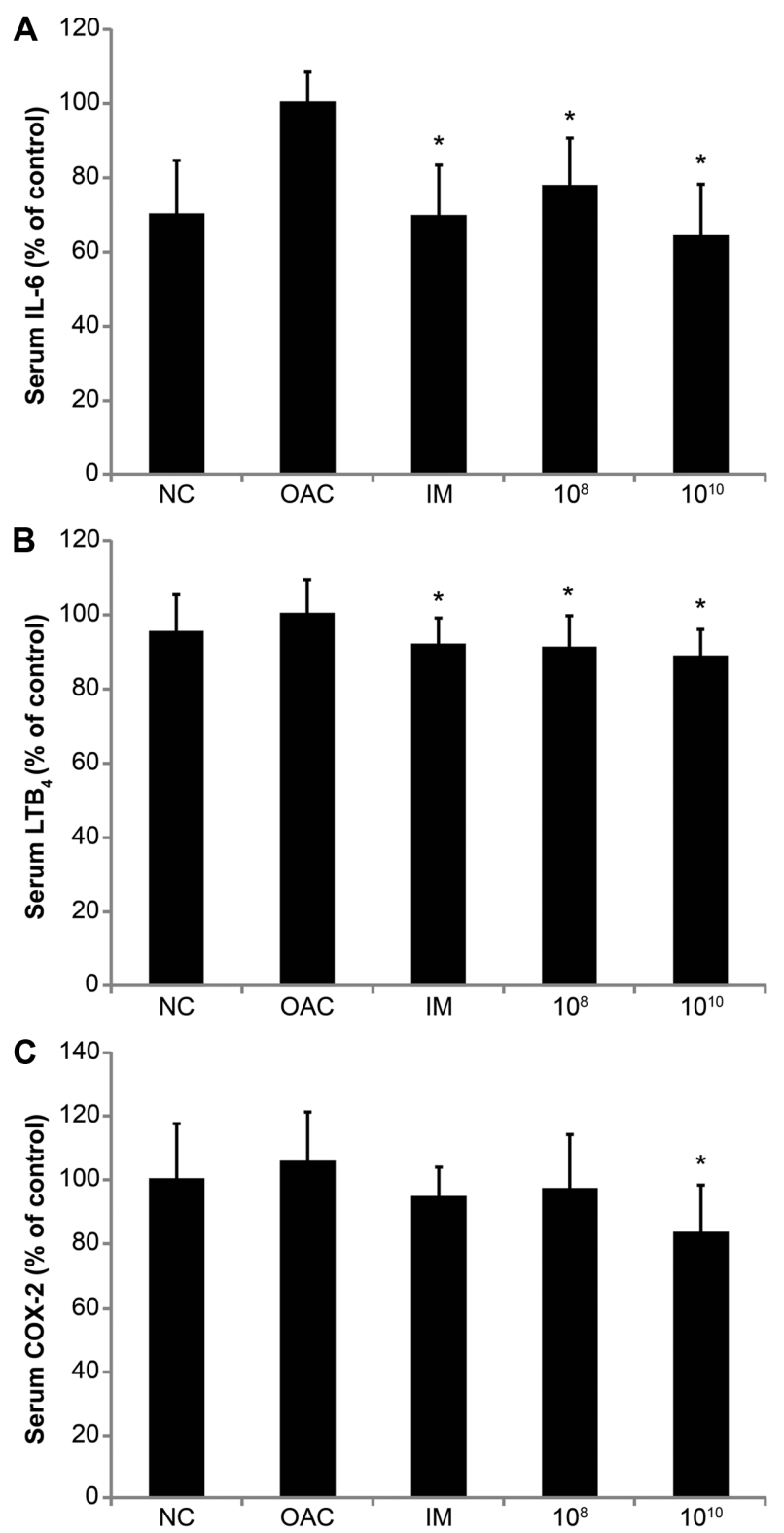

Fig. 1. Effects of ID-CBT5101 on the serum level of inflammatory factors in a monosodium iodoacetate-induced rat model of osteoarthritis.

Luminex and enzyme-linked immunosorbent assays were used to measure the serum levels of interleukin-6 (IL-6) (A), leukotriene B4 $\left(\mathrm{LTB}_{4}\right)(\mathbf{B})$, and prostaglandin-endoperoxide synthase 2 (COX-2) $(\mathrm{C})$. The measured levels were expressed as percent of the control value (mean \pm standard deviation, $n=10$ /group). ${ }^{*} p<0.05$ indicates a significant difference from the osteoarthritis control group (OAC).

that received $10^{10} \mathrm{CFU} /$ day of ID-CBT5101 exhibited significant increases in the IFN- $\gamma$ and GAGs levels (Figs. 2A and $2 \mathrm{~B})$ and a significant decrease in the COMP level $(p<$
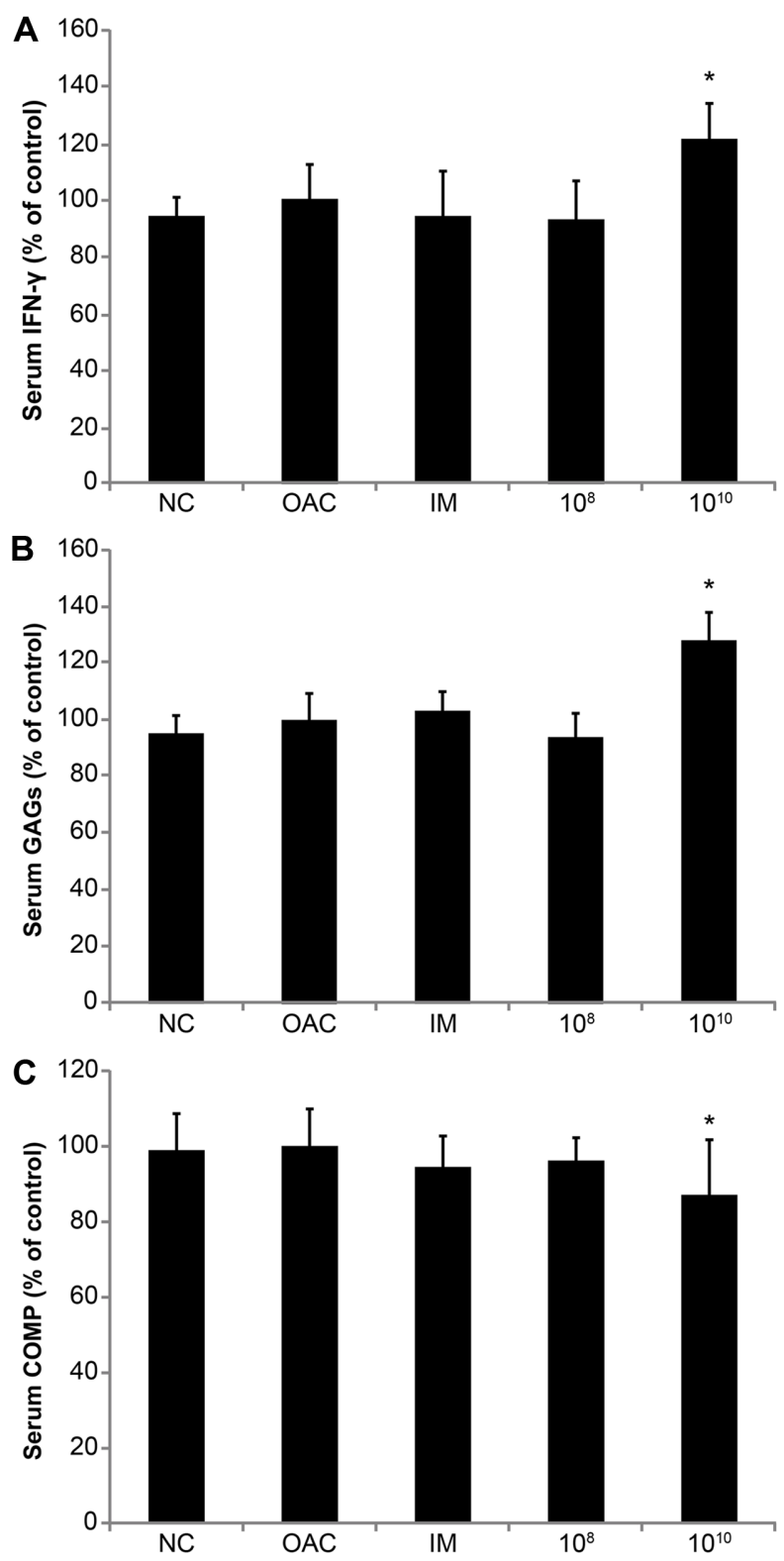

Fig. 2. Effects of ID-CBT5101 on the serum level of bone metabolism factors in a monosodium iodoacetate-induced rat model of osteoarthritis.

Luminex and enzyme-linked immunosorbent assays were used to measure the serum levels of interferon gamma (IFN- $\gamma$ ) (A), glycosaminoglycans (GAGs) (B), and cartilage oligomeric matrix protein (COMP) (C). The measured levels were expressed as percent of the control value (mean \pm standard deviation, $n=10$ /group). ${ }^{*} p<$ 0.05 indicates a significant difference from the osteoarthritis control group (OAC).

0.05) (Fig. 2C). These results indicate that ID-CBT5101 might help protect the cartilage and bone by modulating bone mineral metabolism. 

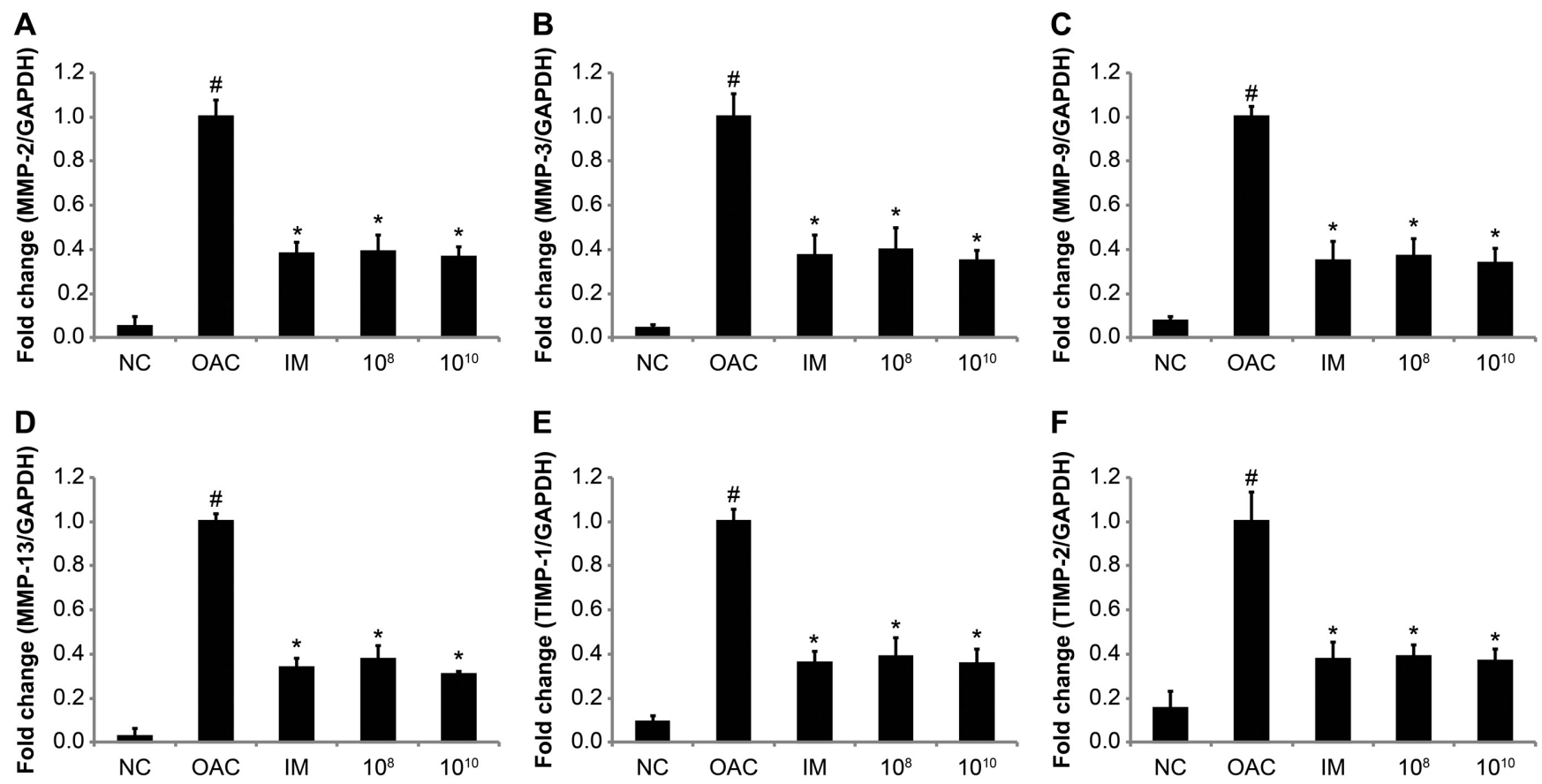

Fig. 3. Effects of ID-CBT5101 on the expression of matrix metalloproteinases and tissue inhibitor of metalloproteinases in the cartilage tissue of a monosodium iodoacetate-induced rat model.

RNA was extracted from the cartilage tissue of rats with osteoarthritis, and the mRNA levels of matrix metalloproteinase-2 (MMP-2) (A), metalloproteinase-3 (MMP-3) (B), metalloproteinase-9 (MMP-9) (C), metalloproteinase-13 (MMP-13) (D), tissue inhibitor of metalloproteinase-1 (TIMP-1) (E), and tissue inhibitor of metalloproteinase-2 (TIMP-2) (F) were determined by the real-time polymerase chain reaction. Glyceraldehyde-3-phosphate dehydrogenase (GAPDH) served as a housekeeping gene. The mRNA expression values are expressed as the mean \pm standard deviation $\left(n=10\right.$ /group). \# $p<0.05$ indicates a significant difference from the normal control group (NC), ${ }^{*} p<0.05$ indicates a significant difference from the osteoarthritis control group (OAC).

\section{Effects of ID-CBT5101 on the Expression of MMPs and TIMPs}

Studies have demonstrated that excessive degradation of type II collagen in arthritic joints is related to increased cleavage by MMPs, which causes irreversible cartilage damage [23]. The MMPs are inhibited by specific TIMPs, and overexpression of MMPs results in an imbalance between the activities of MMPs and TIMPs, which can cause a variety of disorders [24-26]. Compared with the NC group, the OAC group exhibited significantly higher mRNA expression of MMP-2, MMP-3, MMP-9, MMP-13, TIMP-1, and TIMP-2 in the articular cartilage $(p<0.05)$ (Fig. 3). Compared with the OAC group, the ID-CBT5101 and IM treatment groups exhibited significant inhibition $(>60 \%)$ of the expression of these genes $(p<0.05)$ (Figs. 3A-3F). These results confirm that ID-CBT5101 directly controls the expression of MMP, rather than via TIMP stimulation, which suggests that ID-CBT5101 can decrease MMP production and play a crucial role in tissue remodeling, as well as in the destruction of cartilage and bone in the arthritic joint.

\section{Effects of ID-CBT5101 on Changes in the Hind Paw Weight-Bearing Distribution}

The weight-bearing distribution of the sensitized and normal hind limbs was used as a surrogate marker for joint pain [18]. We evaluated the hind paw weight-bearing capacity using an incapacitance tester, and the hind paw weightdistribution ratio was used to assess OA progression [18, 27]. Compared with the NC group, the OAC group exhibited a noticeably lower weigh-distribution ratio. Compared with the OAC group, the group that received $10^{10} \mathrm{CFU} /$ day of ID-CBT5101 exhibited an increase of up to $20 \%$ in the weight-distribution ratio (Fig. 4). These results indicate that ID-CBT5101 might alleviate the clinical symptoms of OA.

\section{Effects of ID-CBT5101 on the OA Histopathology}

We performed H\&E and Safranin O staining of the knee 


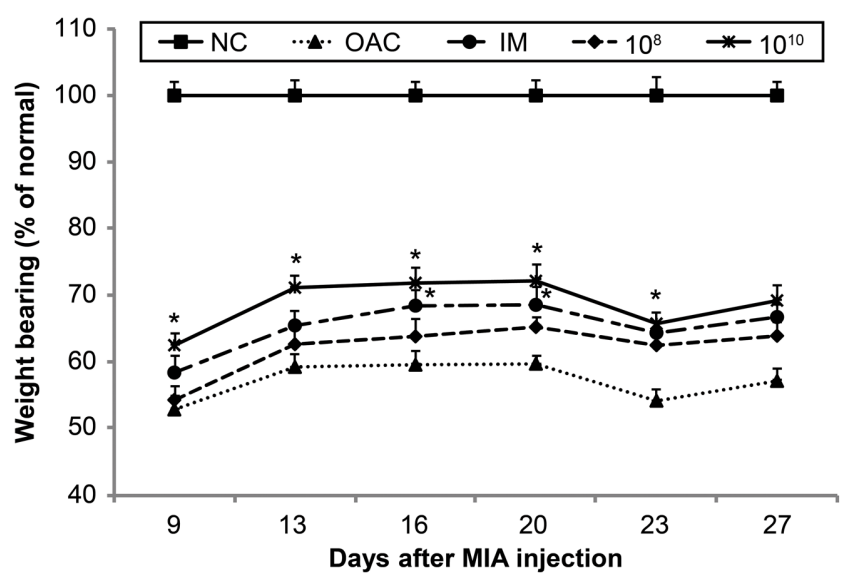

Fig. 4. Effects of ID-CBT5101 on the hind paw weight-bearing distribution in a monosodium iodoacetate (MIA)-induced rat model of osteoarthritis.

Starting on day 9 after MIA injection, the weight-bearing distribution was measured every 4 days using an incapacitance tester. The values are expressed as percent of the normal control (NC) value. (mean \pm standard deviation, $n=10$ /group. ${ }^{*} p<0.05$ indicates a significant difference from the osteoarthritis control group (OAC).

cartilage to determine whether ID-CBT5101 prevented and/or alleviated the histopathology of OA in the knee joint. The H\&E staining results revealed well-preserved cartilage (Fig. 5A, circles), chondroitin sulfuric acid (Fig. 5A, rectangles), and proteoglycan around the cartilage (Fig. 5B, arrows) in the NC group. However, the OAC group exhibited damaged cartilage, increased infiltration of inflammatory cells (Fig. 5A, triangle), and decreased chondroitin sulfuric acid and proteoglycan volumes, with high OARSI scores (Fig. 5C). In contrast, the ID-CBT5101- and IM-treated groups exhibited well-preserved cartilage and proteoglycan volumes, reduced infiltration of inflammatory cells, and increased chondroitin sulfuric acid volume, with significantly lower OARSI scores than those of the OAC group $(p<0.05)$ (Figs. 5A-5C). These results indicate that ID-CBT5101 has anti-inflammatory effects and can protect the knee joint structure.

\section{Effects of ID-CBT5101 on the Femur Morphology and Articular Cartilage Volume}

Decreased articular cartilage volume is the predominant symptom of OA. To determine the effects of ID-CBT5101 on the microarchitecture of knee joints, we performed morphological analysis of the distal femur and articular cartilage using the micro-CT findings. Destruction of the trabecular bone in the distal femur and decreased cartilage volume were confirmed in the OA rats using the 3D micro-
CT images. Compared with the NC group, the OAC group exhibited greater deterioration of the distal femur bone architecture and less cartilage volume (Fig. 6A). The IDCBT5101- and IM-treated groups exhibited a significantly preserved femur bone architecture and significantly greater cartilage volume $(p<0.05)$ (Fig. 6B).

\section{Discussion}

To the best of our knowledge, the present study is the first to provide in vivo evidence that the administration of ID-CBT5101 can prevent and alleviate the progression of $\mathrm{OA}$ in the MIA-induced rat model. These beneficial effects are supported by the findings regarding inflammatory and bone metabolism factors, weight-bearing distribution, and histopathological and morphological characteristics.

The incidence of $\mathrm{OA}$ is increasing owing to prolonged life expectancy, medical developments, and improved standards of living [28]. Patients with OA experience effacement of their articular cartilage that is accompanied by osteoclasia, which are caused by various inflammatory and bone metabolism factors [29]. However, long-term small-molecule treatments (e.g., NSAIDs and COX-2 inhibitors) can cause severe gastrointestinal, renal, and cardiovascular side effects $[30,31]$. Thus, it is important to identify materials or drugs that can prevent and alleviate OA symptoms without adverse effects.

Various inflammatory factors play key roles in the progressive cartilage destruction that is observed in OA cases [32]. For example, COX-2 is involved in inflammation, pain, and cytokine synthesis processes, whereas IL-6 and $\mathrm{LTB}_{4}$ are well-known inflammatory factors that increase bone loss in patients with OA [33-36]. These inflammatory factors are related to the increased COMP levels in the blood, as they activate various matrix metalloproteinases that actively degrade the cartilage (e.g., by removing GAGs) [19-21]. The production of IFN- $\gamma$ is also induced during $\mathrm{OA}$, and this factor can modulate the activity of MMPs [22]. In the present study, ID-CBT5101 reduced the serum levels of inflammatory factors and COMP, and likely protected against OA-related cartilage degradation through the decreased MMP expression in the cartilage. Interestingly, $10^{10} \mathrm{CFU} /$ day of ID-CBT5101 resulted in a greater increase in IFN- $\gamma$ level, compared with that of the IM-treated group. Moreover, the patients with OA may experience pain during joint movements, which typically leads to a diminished use of the joints and reduced joint mobility [37]. Our results indicate that ID-CBT5101 significantly alleviated the weight-bearing distribution in 

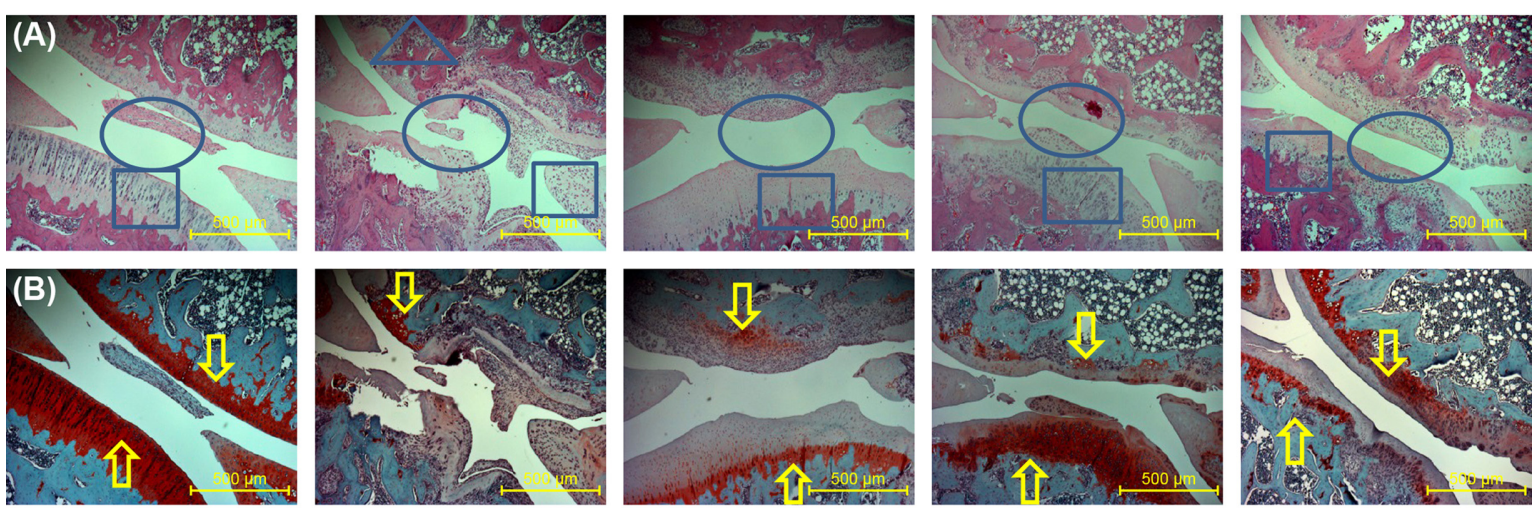

NC

OAC

IM

$10^{8}$

$10^{10}$

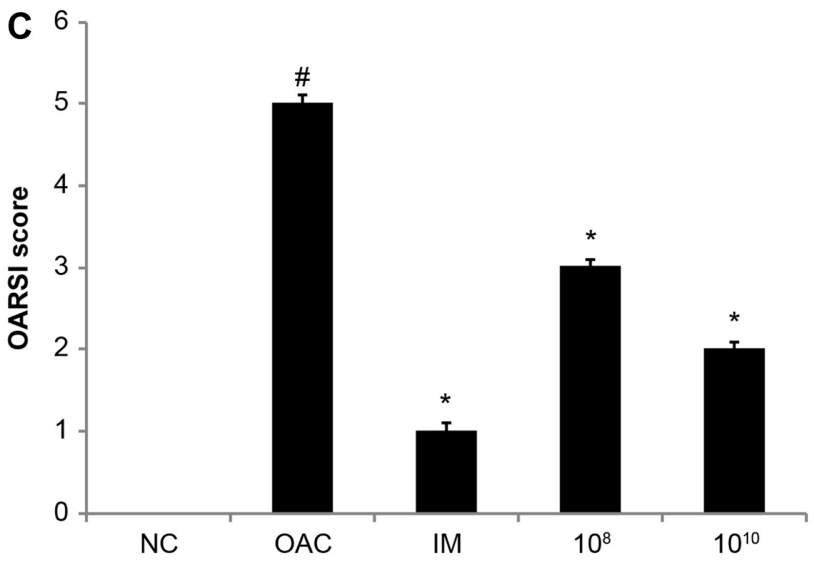

Fig. 5. Histopathological effects of ID-CBT5101 on the knee joints in a monosodium iodoacetate-induced rat model of osteoarthritis.

Representative histological images of knee joint tissues stained using hematoxylin and eosin (A) or Safranin O (B) (magnification, 200x). Osteoarthritis Research Society International (OARSI) scoring was performed by an independent observer (C). \#p $<0.05$ indicates a significant difference from the normal control group (NC), ${ }^{*} p<0.05$ indicates a significant difference from the osteoarthritis control group (OAC).

rats with MIA-induced OA, which suggests that IDCBT5101 might be useful for treating OA pain.

During the onset of $\mathrm{OA}$, the collagen matrix of cartilage becomes disorganized and the proteoglycan volume decreases [38, 39]. Without the protective effects of the proteoglycans, the collagen fibers become more susceptible to degradation, and cartilage damage and loss occur easily, which may also affect other joint structures [40-42]. For example, the ligaments stabilizing the knee joint can become thickened and fibrotic, and the menisci can be damaged and worn away [43]. Furthermore, pain in an osteoarthritic joint may be related to subchondral bone lesions [44]. In the present study, ID-CBT5101 effectively preserved the joint tissue and cartilage, as the ID-CBT5101 treatment groups exhibited minimal OA-related cartilage and skeletal damage, as well as preserved proteoglycan volume. In conclusion, our results indicate that, compared with both negative and positive controls, the ID-CBT5101 treatments exhibited therapeutic effects that decreased the cartilage destruction factors (i.e., inflammation and matrix metalloproteinases). They also protected the cartilage and bone by modulating bone mineral metabolism factors (i.e. IFN- $\gamma$ and glycosaminoglycans). These results have been proven through morphological and histopathological analyses, and the weight-bearing distribution. Therefore, ID-CBT5101 may be a novel therapeutic option to manage OA, although clinical studies are needed to validate these findings in human patients.

\section{Acknowledgments}

This research was supported by the Ildong Pharmaceutical Corporation, and RIC Program of MOTIE (Ministry of Trade, Industry and Energy) in Daejeon University. 


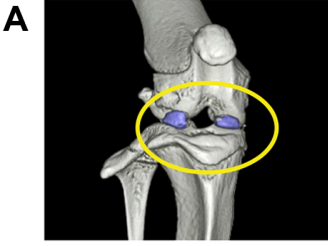

NC

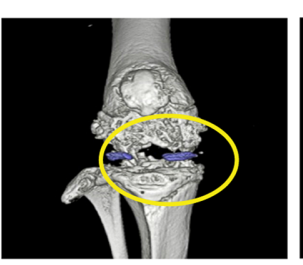

OAC

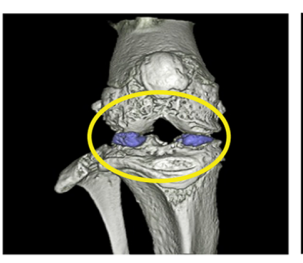

IM

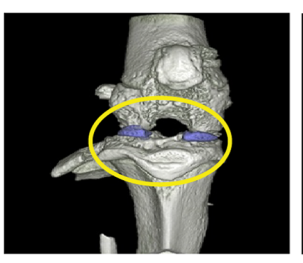

$10^{8}$

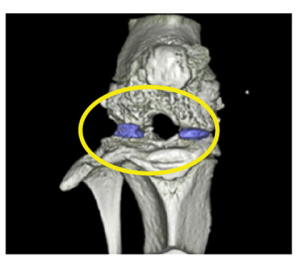

$10^{10}$

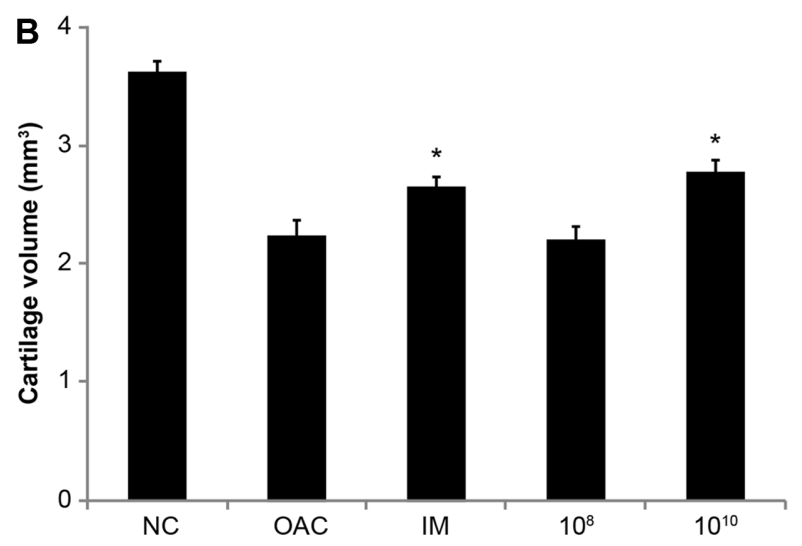

Fig. 6. Effects of ID-CBT5101 on the femur bone architecture and cartilage volume in a monosodium iodoacetate-induced rat model of osteoarthritis.

Representative morphological images of the knee joint tissues were obtained by three-dimension micro-computed tomography (A), and we also measured the corresponding cartilage volume (B). The cartilage volume values are expressed as the mean \pm standard deviation $\left(n=10 /\right.$ group). ${ }^{*} p$ $<0.05$ indicates a significant difference from the osteoarthritis control group (OAC).

\section{Conflict of Interest}

The authors have no financial conflicts of interest to declare.

\section{References}

1. Pritzker KP, Gay S, Jimenez SA, Ostergaard K, Pelletier JP, Revell PA, et al. 2006. Osteoarthritis cartilage histopathology: grading and staging. Osteoarthr. Cartil. 14: 13-29.

2. Longo UG, Loppini M, Fumo C, Rizzello G, Khan WS, Maffulli N, et al. 2012. Osteoarthritis: new insights in animal models. Open Orthop. J. 6: 558-563.

3. Couture RR, Cuello AC. 1984. Studies on the trigeminal antidromic vasodilatation and plasma extravasation in the rat. J. Physiol. 346: 273-285.

4. Brody LT. 2015. Knee osteoarthritis: clinical connections to articular cartilage structure and function. Phys. Ther. Sport 16: 301-316.

5. Park S, Kim YS, Lee D, Kwon Y, Park J, Lee SY, et al. 2014. Efficacy and safety of HT008 and glucosamine sulfate in the treatment of knee osteoarthritis: a randomized double-blind trial. Korean J. Herbol. 29: 45-52.

6. Guzman RE, Evans MG, Bove S, Morenko B, Kilgore K.
2003. Mono-iodoacetate-induced histologic changes in subchondral bone and articular cartilage of rat femorotibial joints: an animal model of osteoarthritis. Toxicol. Pathol. 31: 619-624.

7. Ashraf S, Mapp PI, Burston J, Bennett AJ, Chapman V, Walsh DA. 2014. Augmented pain behavioural responses to intra-articular injection of nerve growth factor in two animal models of osteoarthritis. Ann. Rheum. Dis. 73: 1710-1718.

8. Di Paola R, Fusco R, Impellizzeri D, Cordaro M, Britti D, Morittu VM, et al. 2016. Adelmidrol, in combination with hyaluronic acid, displays increased anti-inflammatory and analgesic effects against monosodium iodoacetate-induced osteoarthritis in rats. Arthritis Res. Ther. 18: 291-302.

9. Fernihough J, Gentry C, Malcangio M, Fox A, Rediske J, Pellas T, et al. 2004. Pain related behaviour in two models of osteoarthritis in the rat knee. Pain 112: 83-93.

10. Puente LD, Betoret NV, Cortes MR. 2009. Evolution of probiotic content and color of apples impregnated with lactic acid bacteria. Vitae 16: 297-303.

11. Abdel-Hafeez HM, Saleh ES, Tawfeek SS, Youssef IM, Abdel-Daim AS. 2016. Effects of probiotic, prebiotic, and synbiotic with and without feed restriction on performance, hematological indices and carcass characteristics of broiler chickens. Asian-Australas. J. Anim. Sci. 30: 672-682.

12. Kumar R, Joshi SR. 2009. Probiotics: biotechnology in 
prolongation of life, pp. 187. In Mishra CS, Champagne P (eds.), Biotechnology Applications. International Publishing House, New Delhi.

13. Vinolo MA, Rodrigues HG, Nachbar RT, Curi R. 2011. Regulation of inflammation by short chain fatty acids. Nutrients 3: 858-876.

14. Vonsy JL, Ghandehari J, Dickenson AH. 2009. Differential analgesic effects of morphine and gabapentin on behavioural measures of pain and disability in a model of osteoarthritis pain in rats. Eur. J. Pain 13: 786-793.

15. McDougall JJ, Watkins L, Li Z. 2006. Vasoactive intestinal peptide (VIP) is a modulator of joint pain in a rat model of osteoarthritis. Pain 123: 98-105.

16. Gerwin N, Bendele AM, Glasson S, Carlson CS. 2010. The OARSI histopathology initiative - recommendations for histological assessments of osteoarthritis in the rat. Osteoarthr. Cartil. 18: S24-S34.

17. Chung HJ, Cho L, Shin JS, Lee J, Ha IH, Park HJ, et al. 2014. Effects of JSOG-6 on protection against bone loss in ovariectomized mice through regulation of osteoblast differentiation and osteoclast formation. BMC Complement. Altern. Med. 14: 184.

18. Chun JM, Kim HS, Lee AY, Kim SH, Kim HK. 2016. Antiinflammatory and antiosteoarthritis effects of Saposhnikovia divaricata ethanol extract: in vitro and in vivo studies. Evid. Based Complement. Alternat. Med. 2016: 1984238.

19. Garnero P, Mazières B, Guéguen A, Abbal M, Berdah L, Lequesne $\mathrm{M}$, et al. 2005. Cross-sectional association of 10 molecular markers of bone, cartilage, and synovium with disease activity and radiological joint damage in patients with hip osteoarthritis: the ECHODIAH cohort. J. Rheumatol. 32: 697-703.

20. Perruccio AV, Chandran V, Power JD, Kapoor M, Mahomed NN, Gandhi R. 2017. Systemic inflammation and painful joint burden in osteoarthritis: a matter of sex? Osteoarthr. Cartil. 25: 53-59.

21. Bellucci F, Meini S, Cucchi P, Catalani C, Nizzardo A, Riva A, et al. 2013. Synovial fluid levels of bradykinin correlate with biochemical markers for cartilage degradation and inflammation in knee osteoarthritis. Osteoarthr. Cartil. 21: 1774-1780.

22. Springall R, Amezcua-Guerra LM, Gonzalez-Pacheco $\mathrm{H}$, Furuzawa-Carballeda J, Gomez-Garcia L, Marquez-Velasco R, et al. 2013. Interferon-gamma increases the ratio of matrix metalloproteinase-9/tissue inhibitor of metalloproteinase-1 in peripheral monocytes from patients with coronary artery disease. PLoS One 8: e72291.

23. Dahlberg L, Billinghurst RC, Manner P, Nelson F, Webb G, Ionescu M, et al. 2000. Selective enhancement of collagenasemediated cleavage of resident type II collagen in cultured osteoarthritic cartilage and arrest with a synthetic inhibitor that spares collagenase 1 (matrix metalloproteinase 1). Arthritis Rheum. 43: 673-682.
24. Yang CC, Lin CY, Wang HS, Lyu SR. 2013. Matrix metalloproteases and tissue inhibitors of metalloproteinases in medial plica and pannus-like tissue contribute to knee osteoarthritis progression. PLoS One 8: e79662.

25. Becher N, Hein M, Uldbjerg N, Danielsen CC. 2008. Balance between matrix metalloproteinases (MMP) and tissue inhibitors of metalloproteinases (TIMP) in the cervical mucus plug estimated by determination of free non-complexed TIMP. Reprod. Biol. Endocrinol. 6: 45.

26. Jeong YJ, Kim I, Cho JH, Park DW, Kwon JE, Jung MW, et al. 2015. Anti-osteoarthritic effects of the Litsea japonica fruit in a rat model of osteoarthritis induced by monosodium iodoacetate. PLoS One 10: $\mathrm{e} 0134856$.

27. Bove SE, Calcaterra SL, Brooker RM, Huber CM, Guzman RE, Juneau PL, et al. 2003. Weight bearing as a measure of disease progression and efficacy of anti-inflammatory compounds in a model of monosodium iodoacetate-induced osteoarthritis. Osteoarthr. Cartil. 11: 821-830.

28. Lunenfeld B, Stratton P. 2013. The clinical consequences of an ageing world and preventive strategies. Best Pract. Res. Clin. Obstet. Gynaecol. 27: 643-659.

29. Redlich K, Smolen JS. 2012. Inflammatory bone loss: pathogenesis and therapeutic intervention. Nat. Rev. Drug Discov. 11: 234-250.

30. Crofford LJ. 2013. Use of NSAIDs in treating patients with arthritis. Arthritis Res. Ther. 15: S2.

31. Lanas A. 2009. Nonsteroidal antiinflammatory drugs and cyclooxygenase inhibition in the gastrointestinal tract: a trip from peptic ulcer to colon cancer. Am. J. Med. Sci. 338: 96-106.

32. Lee SG, Lee EJ, Park WD, Kim JB, Kim EO, Choi SW. 2012. Anti-inflammatory and anti-osteoarthritis effects of fermented Achyranthes japonica Nakai. J. Ethnopharmacol. 142: 634-641.

33. Zhang JM, An J. 2007. Cytokines, inflammation and pain. Int. Anesthesiol. Clin. 45: 27-37.

34. Wittenberg RH, Willburger RE, Kleemeyer KS, Peskar BA. 1993. In vitro release of prostaglandins and leukotrienes from synovial tissue, cartilage, and bone in degenerative joint diseases. Arthritis Rheum. 36: 1444-1450.

35. Kaneko S, Satoh T, Chiba J, Ju C, Inoue K, Kagawa J. 2000. Interleukin-6 and interleukin- 8 levels in serum and synovial fluid of patients with osteoarthritis. Cytokines Cell. Mol. Ther. 6: 71-79.

36. Sokolove J, Lepus CM. 2013. Role of inflammation in the pathogenesis of osteoarthritis: latest findings and interpretations. Ther. Adv. Musculoskelet. Dis. 5: 77-94.

37. Felson DT. 2009. Developments in the clinical understanding of osteoarthritis. Arthritis Res. Ther. 11: 203.

38. Bollet AJ, Nance JL. 1966. Biochemical findings in normal and osteoarthritic articular cartilage. II. Chondroitin sulfate concentration and chain length, water, and ash content. $J$. Clin. Invest. 45: 1170-1177.

39. Brocklehurst R, Bayliss MT, Maroudas A, Coysh HL, Freeman MA, Revell PA, et al. 1984. The composition of 
normal and osteoarthritic articular cartilage from human knee joints. With special reference to unicompartmental replacement and osteotomy of the knee. J. Bone Joint Surg. Am. 66: 95-106.

40. Chou MC, Tsai PH, Huang GS, Lee HS, Lee CH, Lin MH, et al. 2009. Correlation between the MR T2 value at $4.7 \mathrm{~T}$ and relative water content in articular cartilage in experimental osteoarthritis induced by ACL transection. Osteoarthr. Cartil. 17: 441-447.

41. Grushko G, Schneiderman R, Maroudas A. 1989. Some biochemical and biophysical parameters for the study of the pathogenesis of osteoarthritis: a comparison between the processes of ageing and degeneration in human hip cartilage. Connect. Tissue Res. 19: 149-176.

42. Madry H, Luyten FP, Facchini A. 2012. Biological aspects of early osteoarthritis. Knee Surg. Sports Traumatol. Arthrosc. 20: 407-422.

43. Englund M, Roemer FW, Hayashi D, Crema MD, Guermazi A. 2012. Meniscus pathology, osteoarthritis and the treatment controversy. Nat. Rev. Rheumatol. 8: 412-419.

44. Felson DT, Chaisson CE, Hill CL, Totterman SM, Gale ME, Skinner KM, et al. 2001. The association of bone marrow lesions with pain in knee osteoarthritis. Ann. Intern. Med. 134: 541-549. 